\title{
Protein Response Effects on Cofactor Excitation Energies from First Principles: Augmenting Subsystem Time-Dependent Density-Functional Theory with Many-Body Expansion Techniques
}

\section{- Supporting Information -}

\author{
Linus Scholz and Johannes Neugebauer ${ }^{1}$ \\ Theoretische Organische Chemie, Organisch-Chemisches Institut \\ and Center for Multiscale Theory and Computation, \\ Westfälische Wilhelms-Universität Münster \\ Corrensstraße 40, 48149 Münster, Germany
}

Date: $\quad$ September 2, 2021

\footnotetext{
${ }^{1}$ email: j.neugebauer@uni-muenster.de
} 


\section{S1 Green Fluorescent Protein: Small Model}

Table S1: Contributions of individual subsystems (in units of eV) to the total response effect in (embedded) $\operatorname{MBE}(2), \Delta E_{i}^{\operatorname{exc}(2)}$, and (embedded) $\operatorname{MBE}(3), \Delta E_{i}^{\operatorname{exc}(2)}+\Delta E_{i}^{\operatorname{exc}(3)}$, for GFP279-A and -B.

\begin{tabular}{c|cc|cc}
\hline \hline \multirow{2}{*}{ system } & \multicolumn{2}{|c}{ GFP279-A } & \multicolumn{2}{c}{ GFP279-B } \\
& MBE(2) & $\operatorname{MBE}(3)$ & $\operatorname{MBE}(2)$ & $\mathrm{MBE}(3)$ \\
\hline Thr62 & -0.040 & -0.035 & -0.052 & -0.044 \\
\hline Arg96* & -0.054 & -0.054 & -0.051 & -0.052 \\
\hline Tyr145* & -0.011 & -0.010 & -0.034 & -0.025 \\
\hline Thr203* & -0.028 & -0.029 & -0.037 & -0.026 \\
\hline Glu222 & -0.005 & -0.004 & -0.007 & -0.004 \\
\hline water 1 & -0.019 & -0.022 & -0.005 & 0.000 \\
\hline water 2 & -0.006 & -0.005 & -0.004 & -0.004 \\
\hline water 3 & -0.011 & -0.013 & -0.020 & -0.019 \\
\hline water 4 & -0.021 & -0.023 & -0.016 & -0.014 \\
\hline$\sum$ & -0.196 & -0.195 & -0.225 & -0.188 \\
\hline \hline
\end{tabular}




\section{S2 Bos taurus Rhodopsin Model}

Table S2: Contributions of individual subsystems (in units of eV) to the total response effect in $\operatorname{MBE}(2), \Delta E_{i}^{\operatorname{exc}(2)}$, or $\operatorname{MBE}(3), \Delta E_{i}^{\operatorname{exc}(2)}+\Delta E_{i}^{\operatorname{exc}(3)}$, for the bovine rhodopsin model (366 atoms).

\begin{tabular}{c|cc||c|cc}
\hline \hline & MBE(2) & MBE(3) & & MBE $(2)$ & $\operatorname{MBE}(3)$ \\
\hline Gly90 & 0.000 & -0.002 & Met207 & -0.006 & -0.005 \\
\hline Glu113 & +0.011 & +0.009 & Phe208 & -0.002 & -0.001 \\
\hline Gly114 & -0.001 & -0.002 & His211 & -0.001 & 0.000 \\
\hline Ala117 & -0.007 & -0.007 & Phe212 & -0.002 & -0.001 \\
\hline Thr118 & -0.005 & -0.005 & Leu216 & 0.000 & 0.000 \\
\hline Gly121 & -0.002 & -0.001 & Phe261 & -0.004 & -0.002 \\
\hline Glu122 & -0.004 & -0.003 & Trp265 & -0.016 & -0.013 \\
\hline Leu125 & -0.002 & -0.001 & Tyr268 & -0.019 & -0.018 \\
\hline Cys167 & -0.001 & -0.001 & Ala269 & -0.001 & -0.001 \\
\hline Tyr178 & -0.001 & -0.001 & Ala272 & 0.000 & 0.000 \\
\hline Glu181 & -0.001 & -0.006 & Ala292 & -0.002 & -0.002 \\
\hline Ser186 & -0.010 & -0.009 & Phe293 & -0.004 & -0.003 \\
\hline Cys187 & -0.001 & -0.002 & Ala295 & -0.001 & -0.001 \\
\hline Gly188 & -0.002 & -0.002 & water 1 & -0.002 & -0.003 \\
\hline Ile189 & -0.003 & -0.002 & water 2 & -0.001 & -0.004 \\
\hline Tyr191 & -0.005 & -0.006 & $\sum$ & -0.096 & -0.095 \\
\hline \hline
\end{tabular}




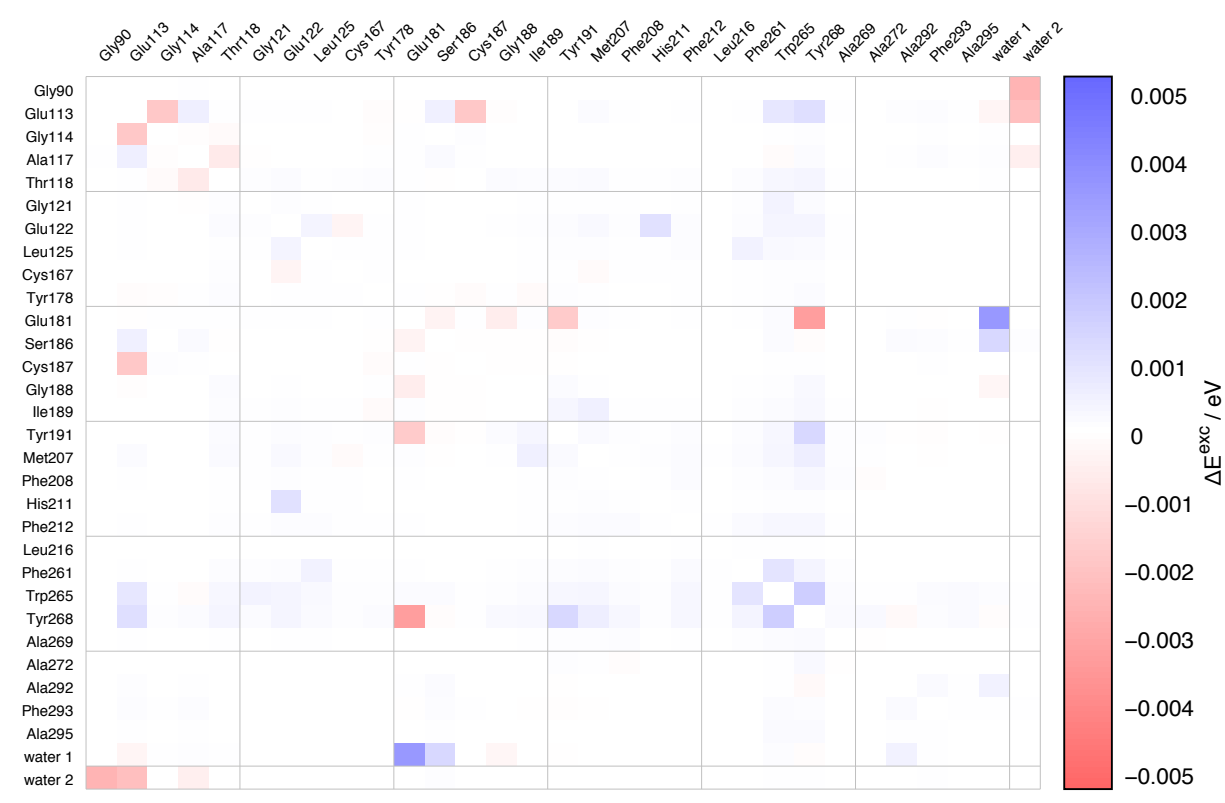

Figure S1: Third-order contributions, $\Delta E_{i j}^{\mathrm{exc}(3)}$, to the chromophore excitation energy of the Bos taurus rhodopsin model after expansion of the chromophore subsystem by two environmental subsystems $i$ and $j$ (rows and columns). Here, calculations include ghost functions for Glu181 and Tyr268 throughout. 
Table S3: Excitation energies $E^{\text {exc }}$ (in units of $\mathrm{eV}$ ) of the many-body expansion and their differences $\left(\Delta_{\text {ref }}\right)$ with respect to the supermolecular reference for the Bos taurus rhodopsin model (366 atoms) including ghost functions for Glu181 and Tyr268 throughout. Data for the isolated chromophore are given for comparison. Furthermore, differences $\left(\Delta_{\text {no-ghost }}\right)$ of the excitation energies to those of the same set of calculations without additional ghost functions are shown ( $c f$. Tab. II).

\begin{tabular}{c|ccc}
\hline \hline \multirow{2}{*}{ system } & \multicolumn{3}{|c}{ rhodopsin } \\
& $E^{\text {exc }}$ & $\Delta_{\text {ref }}$ & $\Delta_{\text {no-ghost }}$ \\
\hline isolated & 2.436 & -0.359 & +0.001 \\
\hline $\operatorname{MBE}(1)$ & 2.886 & +0.090 & +0.001 \\
\hline $\operatorname{MBE}(2)$ & 2.784 & -0.011 & -0.004 \\
\hline $\operatorname{MBE}(3)$ & 2.796 & +0.001 & +0.006 \\
\hline reference & 2.795 & - & - \\
\hline \hline
\end{tabular}


Table S4: Contributions of individual subsystems (in units of eV) to the total response effect in $\operatorname{MBE}(2), \Delta E_{i}^{\operatorname{exc}(2)}$, or $\operatorname{MBE}(3), \Delta E_{i}^{\operatorname{exc}(2)}+\Delta E_{i}^{\operatorname{exc}(3)}$, for the bovine rhodopsin model (366 atoms) including ghost functions for Glu181 and Tyr268 throughout.

\begin{tabular}{l|cc||c|cc}
\hline \hline & MBE(2) & MBE(3) & & $\operatorname{MBE}(2)$ & $\operatorname{MBE}(3)$ \\
\hline Gly90 & 0.000 & -0.002 & Met207 & -0.006 & -0.005 \\
\hline Glu113 & +0.011 & +0.009 & Phe208 & -0.002 & -0.001 \\
\hline Gly114 & -0.001 & -0.002 & His211 & -0.001 & 0.000 \\
\hline Ala117 & -0.007 & -0.007 & Phe212 & -0.002 & -0.001 \\
\hline Thr118 & -0.005 & -0.005 & Leu216 & 0.000 & 0.000 \\
\hline Gly121 & -0.002 & -0.001 & Phe261 & -0.004 & -0.002 \\
\hline Glu122 & -0.004 & -0.003 & Trp265 & -0.016 & -0.013 \\
\hline Leu125 & -0.002 & -0.001 & Tyr268 & -0.020 & -0.018 \\
\hline Cys167 & -0.001 & -0.001 & Ala269 & -0.001 & -0.001 \\
\hline Tyr178 & -0.001 & -0.001 & Ala272 & 0.000 & 0.000 \\
\hline Glu181 & -0.003 & -0.004 & Ala292 & -0.002 & -0.002 \\
\hline Ser186 & -0.010 & -0.009 & Phe293 & -0.004 & -0.003 \\
\hline Cys187 & -0.001 & -0.002 & Ala295 & -0.001 & -0.001 \\
\hline Gly188 & -0.002 & -0.002 & water 1 & -0.003 & -0.001 \\
\hline Ile189 & -0.003 & -0.002 & water 2 & -0.001 & -0.004 \\
\hline Tyr191 & -0.006 & -0.005 & $\sum$ & -0.101 & -0.090 \\
\hline \hline
\end{tabular}




\section{S3 Green Fluorescent Protein: Large Model}

Table S5: Contributions of individual subsystems (in units of $\mathrm{eV}$ ) to the total response effect in $\operatorname{MBE}(2), \Delta E_{i}^{\operatorname{exc}(2)}$, and $\operatorname{MBE}(3), \Delta E_{i}^{\operatorname{exc}(2)}+\Delta E_{i}^{\operatorname{exc}(3)}$, for GFP538-A and -B.

\begin{tabular}{c|cc|cc}
\hline \hline system & \multicolumn{2}{|c}{ GFP538-A } & \multicolumn{2}{c}{ GFP538-B } \\
& MBE(2) & MBE(3) & $\operatorname{MBE}(2)$ & $\operatorname{MBE}(3)$ \\
\hline Leu42 & -0.001 & -0.001 & -0.002 & -0.002 \\
\hline Arg96* & -0.041 & -0.039 & -0.040 & -0.041 \\
\hline Asn121 & -0.001 & -0.001 & -0.002 & -0.001 \\
\hline Tyr145* & -0.014 & -0.012 & -0.033 & -0.026 \\
\hline Phe165* & -0.007 & -0.007 & -0.008 & -0.006 \\
\hline Gln183 & -0.002 & -0.002 & -0.001 & -0.001 \\
\hline Thr203* & -0.023 & -0.024 & -0.033 & -0.024 \\
\hline Glu222 & -0.005 & -0.004 & -0.006 & -0.004 \\
\hline water 1 & -0.019 & -0.021 & -0.003 & 0.000 \\
\hline water 2 & -0.006 & -0.005 & -0.002 & -0.002 \\
\hline water 3 & -0.009 & -0.011 & -0.019 & -0.019 \\
\hline water 4 & -0.012 & -0.012 & -0.010 & -0.009 \\
\hline water 5 & +0.001 & +0.003 & 0.000 & 0.000 \\
\hline water 6 & -0.021 & -0.019 & -0.001 & -0.001 \\
\hline water 7 & -0.001 & -0.001 & -0.001 & -0.001 \\
\hline water 8 & 0.000 & +0.001 & 0.000 & 0.000 \\
\hline$\sum$ & -0.161 & -0.156 & -0.161 & -0.137 \\
\hline \hline
\end{tabular}




\section{S4 Green Fluorescent Protein: Non-embedded MBE for the Small Model}

Table S6: Contributions of individual subsystems (in units of eV) to the total environmental effect in non-embedded $\operatorname{MBE}(2), \Delta E_{i}^{\operatorname{exc}(2)}$, and non-embedded $\operatorname{MBE}(3)$, $\Delta E_{i}^{\operatorname{exc}(2)}+\Delta E_{i}^{\operatorname{exc}(3)}$, for GFP279-A and -B.

\begin{tabular}{c|cc|cc}
\hline \hline \multirow{2}{*}{ system } & \multicolumn{2}{|c}{ GFP279-A } & \multicolumn{2}{c}{ GFP279-B } \\
& $\operatorname{MBE}(2)$ & $\operatorname{MBE}(3)$ & $\operatorname{MBE}(2)$ & $\operatorname{MBE}(3)$ \\
\hline Thr62 & -0.020 & +0.013 & -0.016 & +0.007 \\
\hline Arg96* & -0.130 & -0.122 & -0.009 & -0.120 \\
\hline Tyr145* & -0.007 & -0.009 & +0.003 & +0.025 \\
\hline Thr203* & -0.017 & -0.023 & +0.009 & +0.037 \\
\hline Glu222 & -0.011 & -0.005 & +0.020 & +0.028 \\
\hline water 1 & -0.050 & -0.049 & +0.013 & +0.029 \\
\hline water 2 & -0.005 & +0.006 & -0.009 & +0.005 \\
\hline water 3 & -0.016 & -0.019 & -0.046 & -0.036 \\
\hline water 4 & -0.003 & -0.015 & +0.009 & +0.012 \\
\hline$\sum$ & -0.258 & -0.225 & -0.024 & -0.014 \\
\hline \hline
\end{tabular}



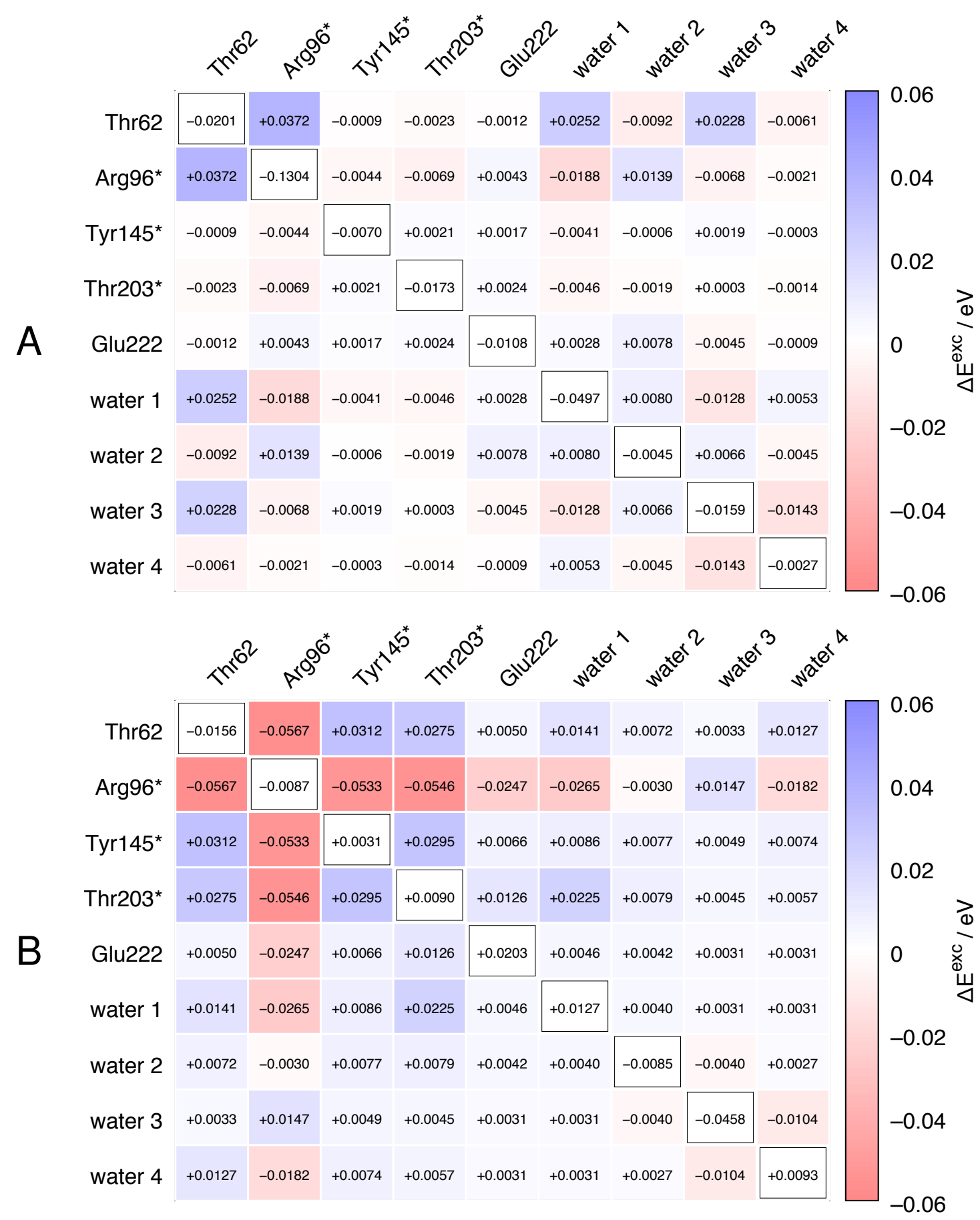

Figure S2: Third-order contributions, $\Delta E_{i j}^{\operatorname{exc}(3)}$, to the chromophore excitation energy in the GFP279 model after expansion of the non-embedded chromophore subsystem by two environmental subsystems $i$ and $j$ (rows and columns). For comparison, the secondorder contributions $\Delta E_{i}^{\operatorname{exc}(2)}$ are shown on the diagonal (note that these are identical to the environmental effects up to second order, $\left.E_{i}^{\operatorname{exc}(2)}-E^{\operatorname{exc}(1)}\right)$. Top: A form, bottom: B form. 


\section{S5 Frontier Orbitals of the Model Systems}

The following figures show isosurface plots (contour value: \pm 0.02 ) of the frontier orbitals (highest occupied molecular orbital, HOMO, and lowest unoccupied molecular orbital, LUMO) of the chromophores for the different model systems studied in the main article. The $\mathrm{HOMO} \longrightarrow$ LUMO orbital transition within the chromophore dominates all electronic excitations investigated in this work. But please note that the isosurface plots were obtained from supermolecular calculations, in order to analyze possible delocalization effects of the orbitals dominantly involved in the electronic transitions. Because of that, the HOMO and LUMO of the chromophore do not necessarily correspond to the HOMO and LUMO of the total system, as indicated in the figure captions. All graphics have been created with VMD. ${ }^{1}$ 


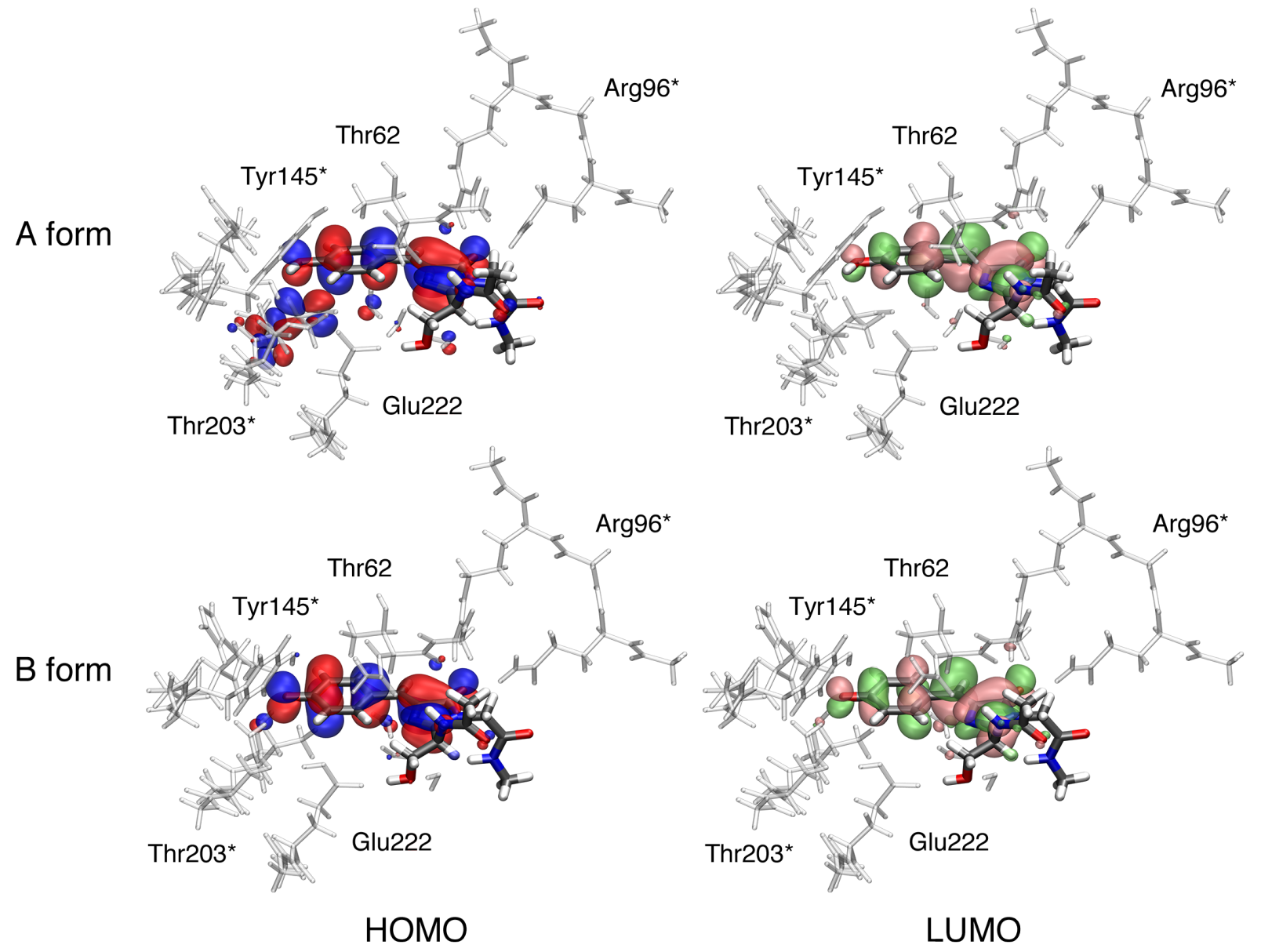

Figure S3: Isosurface plots of the HOMO (left) and LUMO (right) of the GFP279 model in its A form (top) and B form (bottom). 


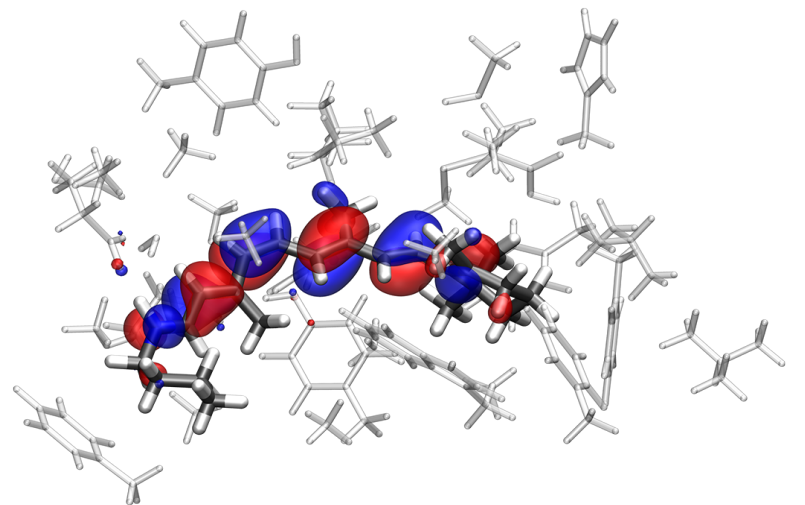

$\mathrm{HOMO}$

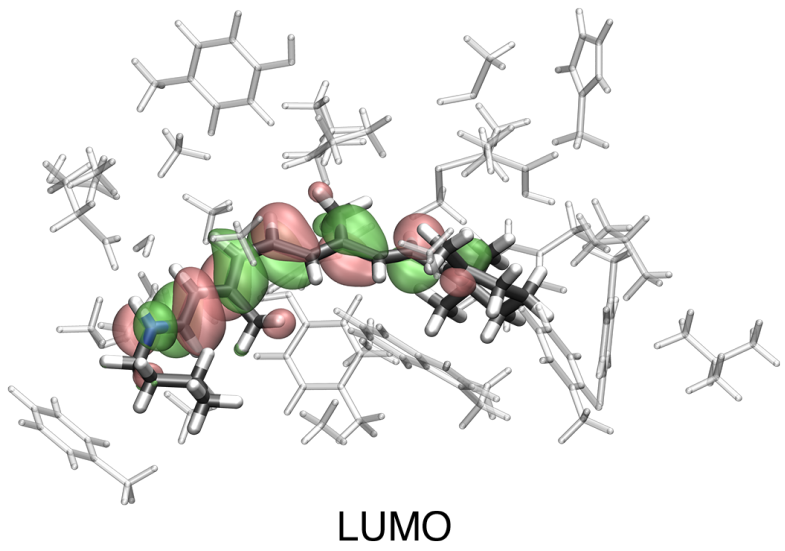

LUMO

Figure S4: Isosurface plots of the HOMO (left) and LUMO (right) of the Bos taurus rhodopsin model. Please note that the labels "HOMO" and "LUMO" refer to the corresponding orbitals of the isolated chromophore. Within the supermolecular calculation, these orbitals appear as $\mathrm{HOMO}-2$ and $\mathrm{LUMO}+2$, respectively. 


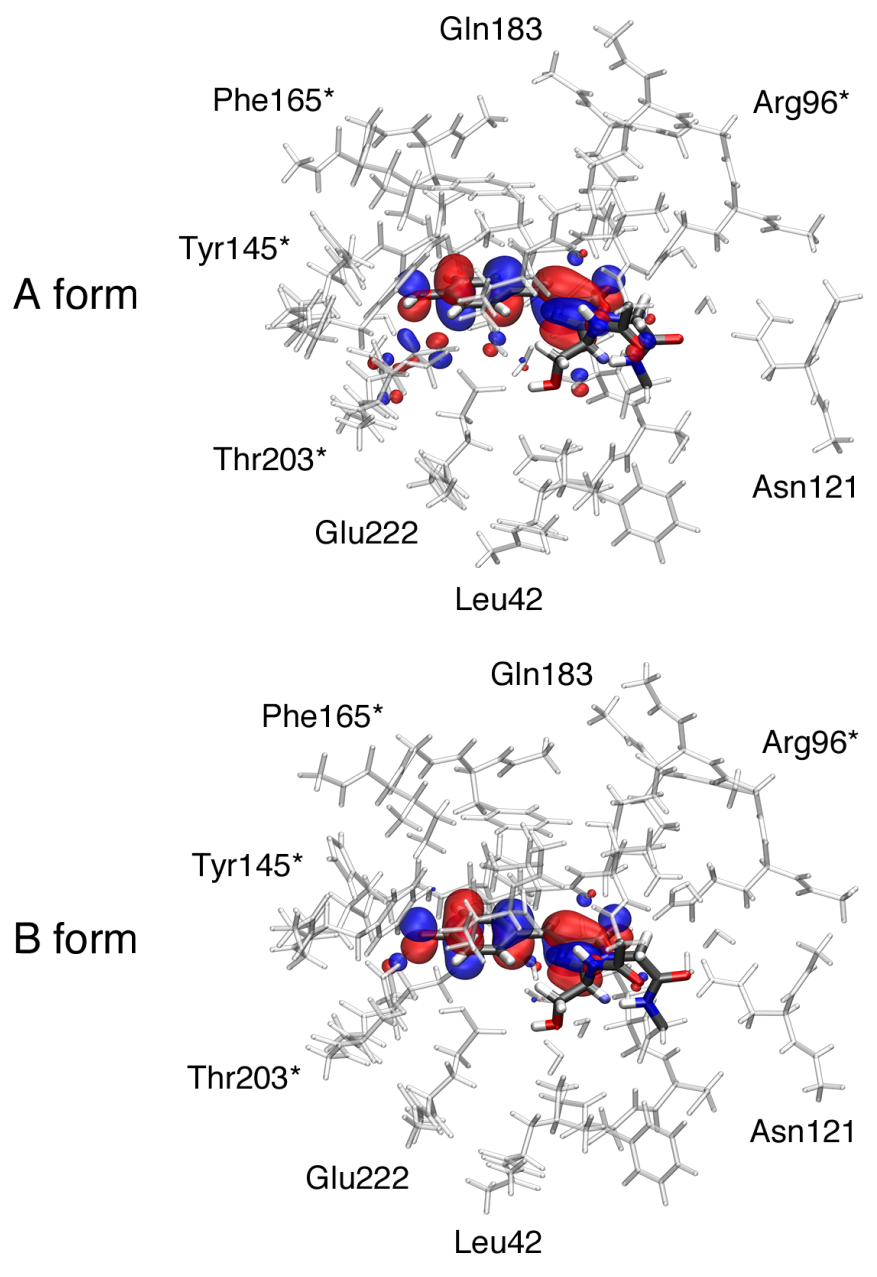

HOMO
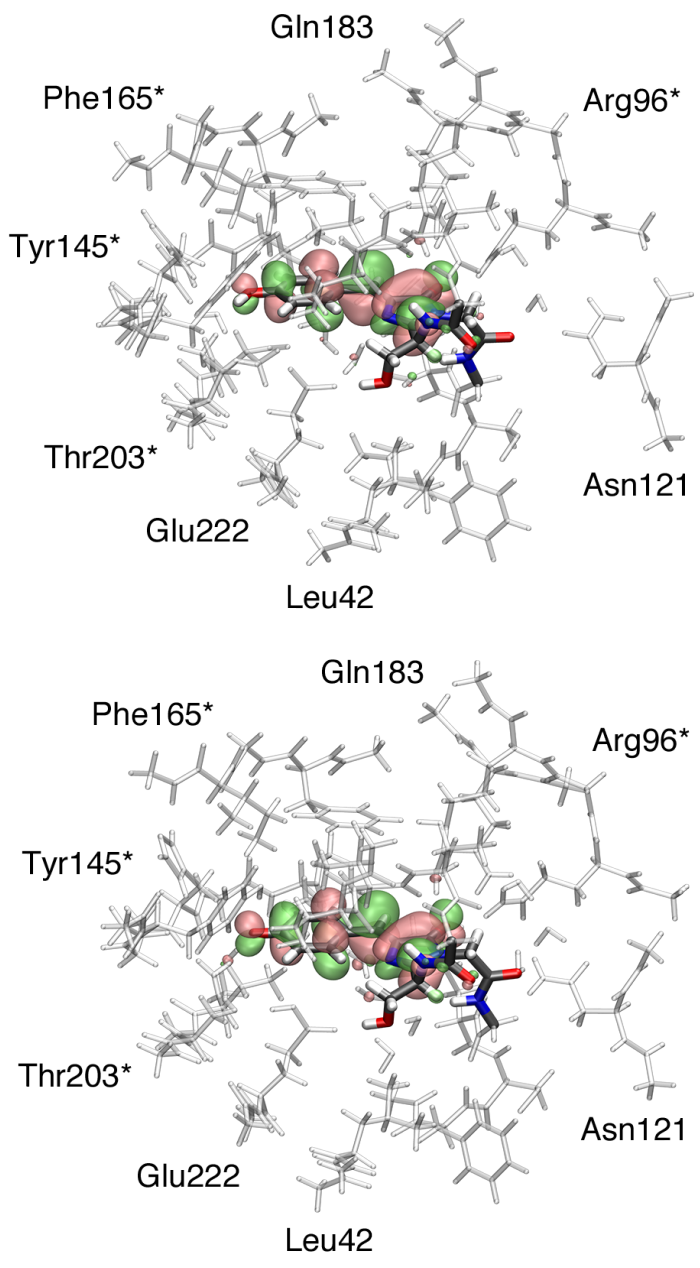

LUMO

Figure S5: Isosurface plots of the HOMO (left) and LUMO (right) of the GFP538 model in its A form (top) and B form (bottom). Please note that the labels "HOMO" and "LUMO" refer to the corresponding orbitals of the isolated chromophore. In case of GFP538-A, the orbital corresponding to the HOMO of the isolated chromophore actually appears as HOMO-5 in the supermolecular calculation. Only those atoms of the chromophore subsystems are highlighted in the licorice representation which are identical to the respective GFP279 model systems. 


\section{References}

[1] Humphrey, W.; Dalke, A.; Schulten, K. VMD - Visual Molecular Dynamics. J. Molec. Graphics 1996, 14, 33-38 